\title{
Ein Beitrag zur Kenntnis des glazial übertieften Inntals westlich von Innsbruck
}

\author{
Von \\ Wilfried Gruber und Franz Weber \\ (Vorgelegt in der math.-nat. Klasse am 9. Oktober 2003 \\ durch das w. M. Franz Weber)
}

\section{Zusammenfassung}

Im Jahre 1991 wurde im Oberen Inntal ein geophysikalisches Messprogramm zur Untersuchung der vermuteten Tiefenrinne abgewickelt. Dabei wurden reflexionsseismische Profile in Völs, Silz, zwei in Oberkofen und je ein refraktionsseismisches Profil in Mils und bei Landeck vermessen. Eine Neubearbeitung der Daten am Profil Völs nutzte intensiv interaktive Auswertealgorithmen und Einzelspuranalysen. Dabei konnte die Datenqualität vor allem innerhalb der ersten $0.3 \mathrm{~s}$ erheblich gesteigert werden.

Es konnten in allen Messgebieten (Silz, Oberhofen, Völs) innerquartäre Reflexionen unterschiedlicher Qualität registriert werden. Regional korrelierbare Leithorizonte fehlen jedoch. Die Form der Übertiefung ist reflexionsseismisch aus den Profilen bei Oberhofen interpretierbar und weist auf eine Muldenform, keineswegs jedoch auf ein U-förmiges Trogtal hin. An den anderen Profilen ist die Quartärbasis reflexionsseismisch nicht als kontinuierlicher Reflektor ausgeprägt, sodass deren Interpretation mehrdeutig ist. Aus der Geschwindigkeitsanalyse, welche jedoch aufgrund der Reflexionsqualität meist nur bis zum Zeitbereich von $0,5 \mathrm{~s}$ verlässliche Aussagen zulässt, wurde der Sprung von ca. 2800 bis $3000 \mathrm{~m} / \mathrm{s}$ als Untergrenze der glazial überkompaktierten Gesteine angenommen. Die Durchschnittsgeschwindigkeit der Sedimente steigt mit der Mächtigkeit der Talfüllung, von $1800 \mathrm{~m} / \mathrm{s}$ bei Landeck auf $2500 \mathrm{~m} / \mathrm{s}$ bei Oberhofen an.

Ein konstruiertes Längsprofil entlang des Oberen Inntals lässt ein starkes Relief des präneogenen Untergrundes vermuten. Von Landeck bis in den Raum östlich Mils erfolgt ein Abfall von $710 \mathrm{~m}$ ü.A. auf ca. $500 \mathrm{~m}$ ü.A., wobei die seismisch homogene 
Talfüllung auf etwa $200 \mathrm{~m}$ Mächtigkeit zunimmt. Westlich der Ötztalmündung wird eine Abtreppung um ca. $150 \mathrm{~m}$ angenommen. Das Profil Silz lässt zwei Möglichkeiten bezüglich der Quartärbasis zu: entweder in ca. $290 \mathrm{~m}$ ü.A. oder in ca. $-10 \mathrm{~m}$ ü.A. Bei Oberhofen wird die Lage der Quartärbasis in $-70 \mathrm{~m}$ ü.A. mit einer Durchschnittsgeschwindigkeit der innertertiären Sedimente von $2500 \mathrm{~m} / \mathrm{s}$ errechnet. Sodann erfolgt nach Osten ein Abfall auf -110 m ü.A. westlich Völs.

Eine seismofazielle Analyse lässt eine weite Verbreitung von glaziolakustrinen und glaziofluviatilen Schichten vermuten. Für Deltabildungen gibt es gewichtige Hinweise, ebenso für das Hereingleiten von Rutschmassen von den Talflanken. Das Vorhandensein von Grundmoränen und Blocklehmen wird aufgrund der Kriterien von Intervallgeschwindigkeit und Reflexionskonfiguration zwar für grundsätzlich möglich erachtet, ist aber derzeit noch hypothetisch.

\begin{abstract}
Geophysical investigations were carried out in 1991 in the Upper Inn Valley to investigate the supposed subsurface channel. The measurements consisted of seismic reflection profiles at Völs and Silz, two profiles at Oberhofen and two seismic refraction profiles at each of Mils and Landeck. The reflection profile at Völs was reprocessed using intensive interactive processes and trace to trace analysis. Especially in the upper time interval, a decided improvement in resolution and data quality was achieved.

Particularly on the seismic reflection profiles, the Quarternary basement cannot be clearly identified and is at any rate not a continuous horizon. The profiles at Oberhofen are an exception. There the topology of the basement is a definite shallow trough rather than a U-shaped valley. Velocity analysis of the rather old data is critical because of the poor data quality, but nevertheless a step in the interval velocities from 2800 to $3000 \mathrm{~m} / \mathrm{s}$ was assumed to define the lower boundary of consolidated glacial rocks. Average velocities of the valley infill increase with thickness from $1800 \mathrm{~m} / \mathrm{s}$ near Landeck to $2500 \mathrm{~m} / \mathrm{s}$ on the Oberhofen Profile.

A profile constructed along the Upper Inn valley shows a powerful relief at the top of the Pre-Neogene rocks. From Landeck to Mils the valley deepens from $710 \mathrm{~m}$ a.s.1. to $500 \mathrm{~m}$, whereas the thickness of the seismically homogenous Neogene rocks increases to $200 \mathrm{~m}$. A step down to $350 \mathrm{~m}$ was suggested west of the Ötztal mouth. The interpretation of the Silz profile gives two possibilities: basement layers either start at $290 \mathrm{~m}$ a.s.l. or at $-10 \mathrm{~m}$. From a depth of $-70 \mathrm{~m}$ at Oberhofen a further drop down to $-110 \mathrm{~m}$ west of Völs was identified.

It can be concluded from Seismic facies analysis, that fluvial and lacustrine glacial rocks form the vast majority of the valley filling. In some cases they were deposited in a deltaic environment or as mass flows. There is strong evidence for consolidated moraines and tills in both the seismic velocities and reflection configuration. However, these layers have not yet been confirmed due to the lack of boreholes.
\end{abstract}

\title{
1. Einleitung und Problemstellung
}

Mit dem Schwerpunkt auf hydrologischer Fragestellung konnte im Jahre 1987 in einer ostalpinen Tallandschaft erstmals eine größere reflexionsseismische Messserie durchgeführt werden. Es wurden in 
fünf getrennten Messgebieten im Inntal sowie im Unterlauf des Zillertals detaillierte reflexionsseismische Messungen als Test für die Anwendbarkeit der Methode unter den gegebenen quartärgeologischen Voraussetzungen ausgeführt. Mit der Grundwasserforschung - insbesondere der tieferen Stockwerke - in einem engen Zusammenhang stehend, konnten auch quartärgeologische Grundfragen bei der Interpretation der Geophysik einbezogen werden, wobei das Problem der Talübertiefung besonders bedeutungsvoll war. Da die reflexionsseismischen Daten in wichtigen Belangen durch eine Tiefbohrung (Wattens 1) überprüft und in geologische Aussagen übersetzt werden konnten, kommt dieser Messkampagne trotz ihres beschränkten Umfanges durchaus Grundsatzcharakter zu. Anfang der Neunziger Jahre wurden auch in der Schweiz analoge reflexionsseismische Messungen in alpinen Tal- und Seelandschaften erfolgreich durchgeführt, sodass die Reflexionsseismik als aussagekräftigstes Messverfahren zur Untersuchung tiefer inneralpiner Täler angesehen werden kann.

Durch das große Interesse der Tiroler Landesregierung als Auftraggeber war es möglich, in den Jahren 1990/91 die Untersuchungen im Oberen Inntal bis Mils, also ca. $50 \mathrm{~km}$ westlich Innsbruck, fortzusetzen. Dabei erfolgte auch methodisch eine Erweiterung, als im Raum Telfs-Innsbruck gravimetrische Messungen als Ergänzung zur Reflexionsseismik durchgeführt wurden. Aus Kostengründen konnten nur schwerpunktförmige Übersichtsmessungen ausgeführt werden.

Durch den Beitritt Österreichs zur EU ist das Inntal zu einem Gebiet von zentraler Bedeutung geworden (Alpentransit, Hochleistungsbahn, Grundwasserreserve). In diesem Zusammenhang ergeben sich neue Fragestellungen, für welche die Kenntnis des Untergrundes wesentlich sind. Bei der Österreichischen Akademie der Wissenschaften wurde vor kurzem ein Forschungsschwerpunkt „Alpenforschung " ins Leben gerufen, bei dessen geowissenschaftlichen Projekten die gegenständlichen Daten nutzbringend verwendet werden können.

\section{Stand der Forschung}

Die Existenz von übertieften inneralpinen Tälern ist heute aufgrund der Erkenntnisse durch Geologie, Tiefbohrungen und Geophysik eine gesicherte Tatsache. Allerdings ist darauf hinzuweisen, dass zum Zeitpunkt der Messkampagne 1987 keine Vergleiche mit anderen Gebieten innerhalb der Ost- und Westalpen verfügbar waren, sodass mit den Untersuchungen durchaus Neuland betreten wurde. Wertvolle 
Vorarbeiten zum geophysikalischen Verständnis wurden durch die quartärgeologischen Arbeiten am Alpennordrand beigetragen (BADER, 1979; GRAF, 1970; SCHNEIDER et al., 1987; VIDAL, 1979; WILDI, 1984); in Österreich hat die Arbeit von VAN HUSEN (1979) Grundlagencharakter. Für das Inntal stand von vornherein fest, dass es aufgrund der Position als inneralpines Neogenbecken und der Beckengeometrie nicht ohne weiteres mit den Zungenbecken wie Rheintal oder den südalpinen Becken verglichen werden konnte. Daher mussten auch die Ergebnisse der seeseismischen Messungen im Bodensee und den Schweizer Seen (SCHOOP und WEGENER, 1984; FINKH et al., 1984) als nicht übertragbar betrachtet werden.

Namhafte geophysikalische Beiträge wurden anfangs auch von der Gravimetrie beigebracht. WALACH (1990), WALACH und POSCH (1989) konnten die Aussagemöglichkeiten spezieller gravimetrischer Methoden (Restschwere, Vertikal- und Horizontalgradient) in Vorarlberg testen und darlegen, dass durch die Modellierung unter Annahme petrophysikalisch vernünftiger Daten eine brauchbare Tiefenberechnung und Erfassung des Untergrundsreliefs möglich ist. Die Arbeit von KISSLING und SCHWENDENER (1990) bestätigte den Wert gravimetrischer Messungen für die Schweizer quartärgeologischen Probleme, wobei erwähnenswert ist, dass selbst mit einem Zweischichtmodell realistische, mit seismischen Daten gut übereinstimmende Tiefenwerte erlangt werden konnten.

Erste geophysikalische Hinweise für Talübertiefungen wurden von ARIC und STEINHAUSER (1976) im Inntalprofil von Thaur gegeben, wo reflexions- und refraktionsseismische Messungen und Gravimetrie zum Einsatz kamen. Durch die gravimetrische Störkörperberechnung und Modellierung konnte am nördlichen Talrand eine ca. $1 \mathrm{~km}$ breite und maximal $700 \mathrm{~m}$ tiefe, asymmetrische Muldenzone nachgewiesen werden. Das reflexionsseismische Profil erstreckte sich vom Inn bis Thaur größtenteils außerhalb der Eintiefung bei Thaur; es konnte jedoch die Lage des präquartären Untergrundes in ca. 350 bis $390 \mathrm{~m}$ Tiefe durch Vergleich mit dem Refraktor (Geschwindigkeit $4000 \mathrm{~m} / \mathrm{s}$ ) im Großteil des Profiles eindeutig festgelegt werden. Für das Quartär wurde eine Durchschnittsgeschwindigkeit von $2000 \mathrm{~m} / \mathrm{s}$ angenommen, stark konsolidierte Sedimente mit Geschwindigkeiten über $3000 \mathrm{~m} / \mathrm{s}$ konnten nicht nachgewiesen werden.

Bei einer ersten Interpretation reflexionsseismischer Daten im Raum Wattens wurde ein höherer Reflektor als Quartärbasis angenommen, der erst durch das Sonic-log in der Tiefbohrung Wattens 1 als Oberkante überkompaktierter Schichten mit Geschwindigkeiten von $4000 \mathrm{~m} / \mathrm{s}$ erkannt wurde. 
Wichtige Erkenntnisse brachten die Untersuchungen in den Schweizer Tälern, wobei die Messungen im Rhone- und Rheintal eher Vergleiche mit dem Inntal zulassen, während bei den Ergebnissen in der Ticino-Region die unterschiedliche geologische Geschichte und die hydrographischen Verhältnisse zu berücksichtigen sind. Diese Arbeiten brachten auch einen Fortschritt bei der reflexionsseismischen Interpretation, da wichtige seismofazielle Sequenzen durch Bohrungen getestet und durch seismisches Modellieren das Verständnis für die zugrundeliegende Geologie gefördert wurde (FINCKH und FREI, 1991; PFIFFNER et al., 1997).

\section{Reflexionsseismik}

\subsection{Datengewinnung}

Der primäre Anlass für das reflexionsseismische Messprogramm lag in hydrogeologischen Fragestellungen. Da tiefere Bohrungen fehlten, ging es zunächst darum, Beiträge für eine Beurteilung verschiedener Gebiete hinsichtlich der Möglichkeit des Auftretens tieferer Grundwasserstockwerke zu schaffen. Aus diesem Grund wurde eine analoge Vorgangsweise wie bei den Arbeiten des Jahres 1987 im Unteren Inntal (WEBER et al., 1990) gewählt, indem der Raum zwischen Innsbruck und Mils (westlich Imst) mit drei reflexionsseismischen Längsprofilen, einem Querprofil und einer refraktionsseismischen Linie untersucht wurde (Abb. 1). Diese Vorgangsweise weicht gezwungenermaßen von der Strategie bei der Prospektion eines Gebietes ab, wo zunächst weiträumige Übersichtsmessungen

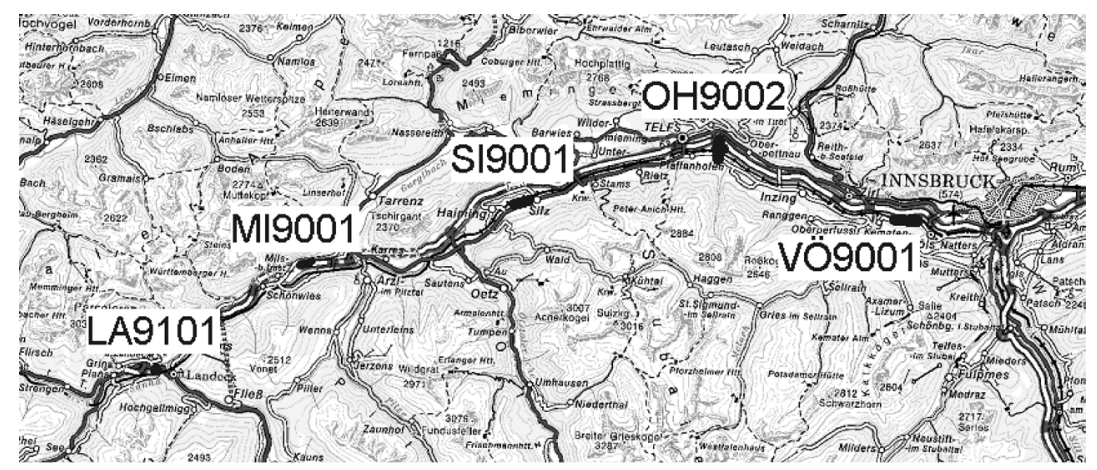

Abb. 1. Lageplan der Profile 
Tabelle 1. Feldparameter der Seismiklinien

\begin{tabular}{ll}
\hline Überdeckung & 12-fach \\
Spread & Mittelpunktaufstellung mit einem Fenster von 4 Spuren \\
Pattern & 16 Geophone pro Spur mit einem Geophonpattern von $15 \mathrm{~m}$ \\
Stationsabstand & $10 \mathrm{~m}$
\end{tabular}

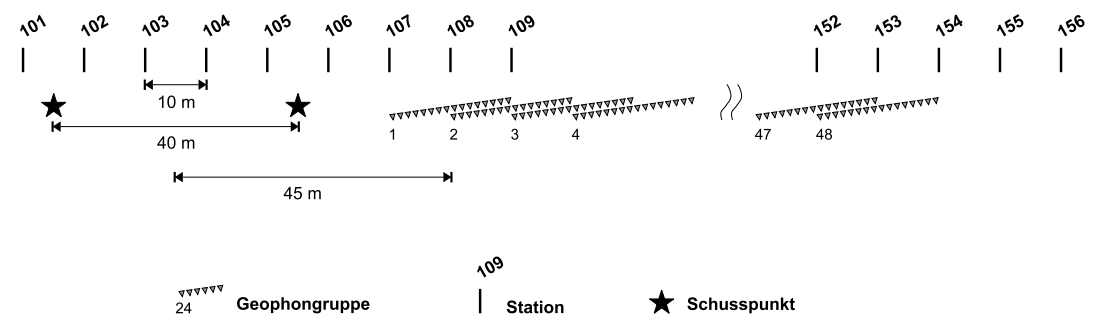

Abb. 2. Aufnahmegeometrie der reflexionsseismischen Profile

Tabelle 2. Daten der Seismikprofile im Untersuchungsgebiet

\begin{tabular}{lclrl}
\hline Profil & Anzahl der Records & Überdeckung & Länge & Methode \\
\hline VOE9001 & 115 & 18 & $1750 \mathrm{~m}$ & Reflexion \\
SI9001 & 60 & max. 18, im Mittel 12 & $1310 \mathrm{~m}$ & Reflexion \\
OH9001 & 86 & 18 & $1530 \mathrm{~m}$ & Reflexion \\
OH9002 & 57 & max. 44, im Mittel 20 & $590 \mathrm{~m}$ & Reflexion \\
Mils & & 2-fach & $1075 \mathrm{~m}$ & Refraktion \\
\hline
\end{tabular}

und anschließend in besonders günstigen Gebieten Detailmessungen ausgeführt werden.

Die Registrierung erfolgte mit einer 48-kanäligen digitalen reflexionsseismischen Apparatur der Type SERCEL 338 B. Die zugrundeliegenden Feldparameter sind in Tabelle 1 aufgelistet, das Akquisitionsschema ist in Abb. 2 ersichtlich. Es wurden fünf seismische Profile vermessen, deren Detaildaten in Tabelle 2 zusammengestellt sind. Die Aufnahmegeometrie richtete sich nach den Möglichkeiten der verwendeten Seismikapparatur. Es galt, die vorhandenen 48 Kanäle für eine maximal mögliche Eindringtiefe so anzuordnen, dass auch noch im seichten Bereich eine vernünftig große laterale Auflösung erzielt werden konnte. Die einzelnen Geophongruppen zu je 16 Einzelgeophonen wurden in einem Pattern mit $20 \mathrm{~m}$ Länge im Abstand von $10 \mathrm{~m}$ ausgelegt. 
Aus der in Abb. 2 gezeigten Geometrie ergibt sich für jede Einzelaufnahme eine gleichmäßige Offsetverteilung zwischen 45 und $515 \mathrm{~m}$. Nach jedem Schuss wurde die Aufstellung um zwei Stationen weiter verschoben und nach jedem sechsten Schuss ein zusätzlicher Gegenschuss (end-off) ausgeführt. Daraus errechnet sich eine nominelle Überdeckung von 14 für ein CDP-gather.

Die Energieerzeugung erfolgte mit Sprengladungen von 0,15 bis $0,21 \mathrm{~kg}$, aufgeteilt auf meist zwei $1,2 \mathrm{~m}$ tiefe im Abstand von $40 \mathrm{~m}$ gelegene Schusslöcher. Diese relativ kleine Sprengladung war vornehmlich aus Umweltschutzgründen erforderlich, da die Bevölkerung gegen die notwendigen Bodeneingriffe sehr sensibilisiert war. Weiters konnten dadurch die störenden Oberflächenwellen auf ein Minimum reduziert und der hochfrequente Anteil der Reflexionsenergie gewahrt werden. Da bereits bei den ersten Messungen zu erkennen war, dass die Reflexionsqualität ungünstiger als bei früheren Untersuchungen im Unteren Inntal war, wurde zum teilweisen Ausgleich ein größerer Überdeckungsgrad gewählt, als ursprünglich die Absicht war.

\subsection{Datenbearbeitung}

Bezüglich der Einzelheiten des Processings der Erstausarbeitung 1991 darf auf frühere Arbeiten verwiesen werden (WEBER et al., 1990, 1991), sowie auf Tabelle 3, in der auch die Erfahrungen der letzten Jahre ihren Niederschlag finden. Im Folgenden soll besonderes Augenmerk auf die Möglichkeiten im Zuge der Neubearbeitung der Linie Völs gelegt werden.

Der Begriff „Reprocessing “ implementiert, dass an bestehenden, in früherer Zeit ausgearbeiteten Datensätzen entweder neue oder ergänzende Bearbeitungsschritte zur Steigerung der Qualität und damit der Interpretierbarkeit durchgeführt werden. Im gegenständlichen Fall wurde eine komplette Neuauswertung ausgehend von den unbearbeiteten Originalaufnahmen durchgeführt. Das bietet den Vorteil, dass in jedem einzelnen Auswerteschritt alternative Bearbeitungssequenzen getestet und bei entsprechendem Ergebnis angewendet werden können. Als Zielvorgabe, die es zu übertreffen galt, lag die reflexionsseismische Auswertung aus dem Jahr 1990 vor (Abb. 6).

In der Prestackphase des Processings war der erste qualitätsentscheidende Schritt das Editieren der demultiplexten Daten. Entgegen früherer Vorgangsweisen, wo fehlerhafte Spuren auf Rohdatenplots identifiziert wurden, stehen heute interaktive Werkzeuge zur 
Tabelle 3. Gegenüberstellung der Auswerteparameter aus dem Jahr 1990 und dem Jahr 2003

\begin{tabular}{|c|c|c|}
\hline Bearbeitungsschritt & Auswertung 1990 & Auswertung 2003 \\
\hline \multicolumn{3}{|l|}{ Prestack: } \\
\hline \multicolumn{3}{|l|}{ Demultiplexen } \\
\hline Edit & optisch von Hand & Interaktiv \\
\hline \multicolumn{3}{|l|}{ Störungsunterdrückung: } \\
\hline Kerbfilter & - & $16,67 \mathrm{~Hz}$ \\
\hline Störfrequenzglättung & - & $50 \mathrm{~Hz}$ \\
\hline \multirow[t]{2}{*}{ Divergenzkorrektur } & exponentiell nach RMS & exponentiell nach RMS \\
\hline & Geschwindigkeit & Geschwindigkeit \\
\hline Ersteinsatzmute & aggressiv, von Hand & konservativ, interaktiv \\
\hline \multicolumn{3}{|l|}{ Waveletprocessing: } \\
\hline Spectral Whitening & 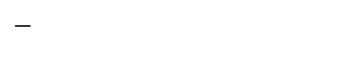 & $\begin{array}{l}\text { nullphasiger Operator, } \\
25-110 \mathrm{~Hz}\end{array}$ \\
\hline Deconvolution & $\begin{array}{l}4 \text { Designfenster, } \\
\text { Mehrkanal- } \\
\text { Spikeoperator }\end{array}$ & $\begin{array}{l}4 \text { Designfenster, } \\
\text { Mehrkanal- } \\
\text { Spikeoperator }\end{array}$ \\
\hline \multicolumn{3}{|l|}{ Sortieren in CMP Ordnung } \\
\hline Geschwindigkeitsanalyse & CVA auf Papier & interaktiv \\
\hline Dynamische Korrektur & ohne stretch mute & $10 \%$ stretch mute \\
\hline Statische Korrekturen: & $\begin{array}{l}\text { Refraktionsauswertung } \\
\text { nach Palmer }\end{array}$ & $\begin{array}{l}\text { interaktive } \\
\text { Refraktionsauswertung } \\
\text { nach Palmer }\end{array}$ \\
\hline $\begin{array}{l}\text { Kurzwellenstatische Korr. } \\
\text { Datumstatische Korr. }\end{array}$ & - & $\begin{array}{l}470 \mathrm{~m} \text { Wellenlängenfilter } \\
\text { auf } 570 \mathrm{~m}\end{array}$ \\
\hline Reststatische Korrektur & ja & ja \\
\hline Stapelung & $\begin{array}{l}\text { normalisiert nach } \\
\text { Sampleanzahl }\end{array}$ & $\begin{array}{l}\text { normalisiert nach } \\
\text { Sampleanzahl }\end{array}$ \\
\hline \multicolumn{3}{|l|}{ Poststack: } \\
\hline Fk-Filter & - & Pass $\pm 13 \mathrm{~ms} /$ Spur \\
\hline Bandpass Filter & $\begin{array}{l}0-700 \mathrm{~ms}: 20-80 \mathrm{~Hz} \\
2000-3000 \mathrm{~ms}: 20-60 \mathrm{~Hz}\end{array}$ & $\begin{array}{l}0-150 \mathrm{~ms}: 20-110 \mathrm{~Hz} \\
200-400 \mathrm{~ms}: 18-80 \mathrm{~Hz} \\
700-1500 \mathrm{~ms}: 15-60 \mathrm{~Hz}\end{array}$ \\
\hline Kohärenzfilter & - & ja \\
\hline
\end{tabular}

Verfügung. Entscheidend war, dass es die geringe Datenmenge erlaubte, jede einzelne Spur auf ihre Qualität zu kontrollieren und wenn notwendig vollständig oder teilweise zu eliminieren. Dadurch wurde es auch möglich, den Einflussbereich der entlang des Profils variierenden Störquellen genau zu definieren, um gezielte, entgegenwirkende Processingschritte an einzelnen Abschnitten anzuwenden. Beispielsweise verursachte die naheliegende Bahntrasse im östlichen Profilabschnitt monofrequente Störungen mit $162 / 3 \mathrm{~Hz}$, welche dort 

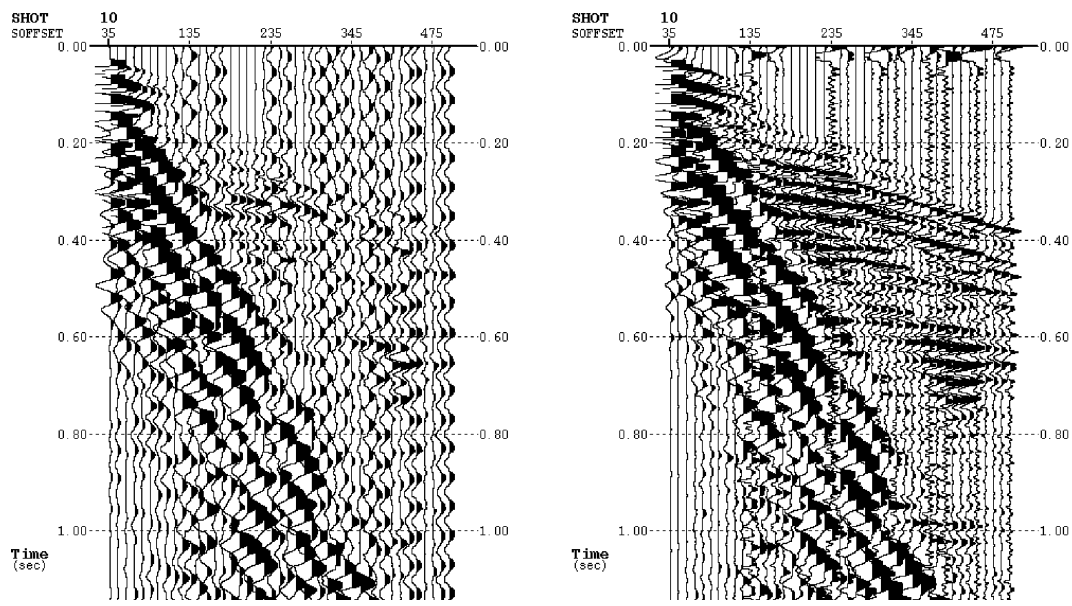

Abb. 3. Verbesserung der Reflexionsqualität an Schuss Nr. 10 durch gezielte Störungsunterdrückung

zusätzlich durch die kreuzende Stromfreileitung mit einem $50 \mathrm{~Hz}$ Signal überlagert wurden. In Abb. 3 ist der Erfolg des Kerbfilters gegen die niedrigere Störfrequenz dokumentiert. Im Bereich von $50 \mathrm{~Hz}$ ist ein Filter insofern kritisch, weil dadurch das Nutzsignal beeinträchtigt wird. Qualitätsverbesserung wurde jedoch mit einer lokalen Glättung des Amplitudenspektrums erreicht.

Besondere Bedeutung bei der Qualität der seicht liegenden Reflexionen kommt dem Muting der Ersteinsätze zu. Im Gegensatz zur Erstauswertung 1991 wurde dies im Reprocessing interaktiv erst nach der Störungsunterdrückung und der Anwendung des kurzwelligen Anteils der statischen Korrekturen durchgeführt. Dabei war die Strategie so ausgelegt, dass bei geringen Offsets eher zu wenige als zu viele Daten möglichst knapp nach dem Ersteinsatz entfernt wurden.

Der entscheidendste Beitrag zur Steigerung der Auflösung wurde im Waveletprocessing erreicht. $\mathrm{Zu}$ deren optimaler Ausführung wurde eine eingehende Testphase der einzelnen Parameter vorgeschaltet. An den Daten wurde zunächst ein Spectral-Whitening durchgeführt und anschließend eine Spikedekonvolution. Das folgt den Grundsätzen von YILMAZ (1987), welcher deterministische Operationen und solche, die dem Spreading entgegenwirken, vor einer statistischen Dekonvolution anwendet. Die im Vergleich zur ersten Auswertung erweiterte Bandbreite ist in Abb. 4 gegenübergestellt. 


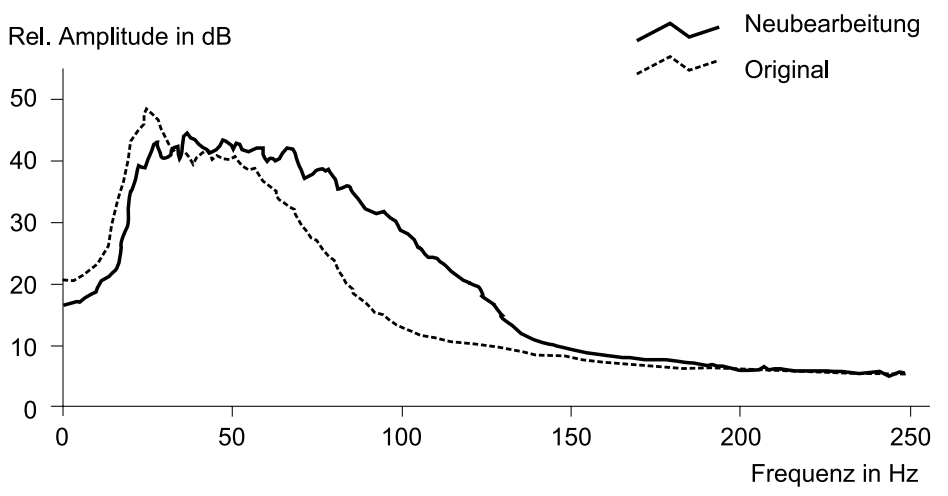

Abb. 4. Normiertes Amplitudenspektrum der Zeitsektion $(0-800 \mathrm{~ms})$ vor und nach der Neubearbeitung

Die Geschwindigkeiten für die dynamische Korrektur wurden in mehreren Iterationen interaktiv ermittelt, wobei auf ein konsistentes Bild der seismischen Geschwindigkeiten im gesamten Messgebiet geachtet wurde. Erst nach der dynamischen Korrektur erfolgte die Anwendung von datumstatischen Korrekturen. Das Bezugsniveau konnte entgegen der Auswertung von 1991 um 20 m näher an die Oberfläche gelegt werden. Dadurch blieben Informationen aus geringer Tiefe weitgehend im Seismogramm erhalten.

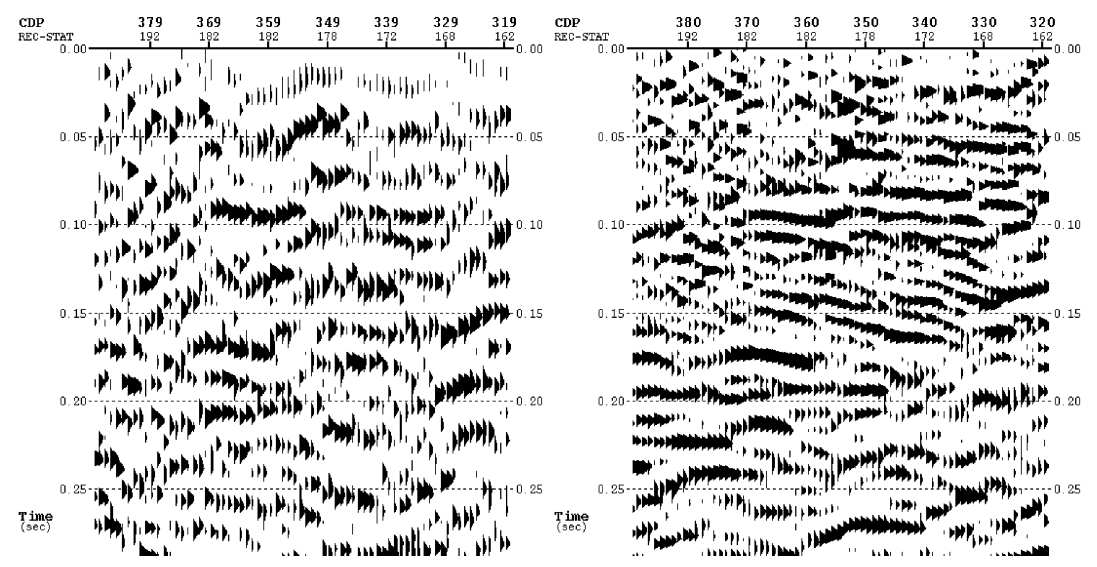

Abb. 5. Gegenüberstellung eines Ausschnitts aus dem seichten Bereich zwischen Station 160 und 190 
Im Poststackprocessing konnte dem gesteigerten Frequenzinhalt Rechnung getragen werden. So wurde die obere Sperrfrequenz im Bandpassfilter von $80 \mathrm{~Hz}$ auf $110 \mathrm{~Hz}$ erhöht, ohne die Sektion durch Hintergrundrauschen zu beeinträchtigen. Die Endabspielung der neu bearbeiteten Linie Völs ist in Abb. 7 dargestellt. Die Steigerung der Auflösung und der Interpretierbarkeit im Gegensatz zur Auswertung 1991 wird durch einen Ausschnitt in Abb. 5 vermittelt.

\section{Interpretation}

\subsection{Allgemeines}

Die Interpretation der zunächst in Zeitsektionen dargestellten reflexionsseismischen Profile beinhaltet folgende Aufgabenstellungen:

- Strukturelle Interpretation

- Seismofazielle Interpretation

- Lithologieerkennung

Ein grundlegendes Informationsdefizit einer reflexionsseismischen Sektion ist ihr vertikaler Maßstab als Zweiweglaufzeit und nicht, wie vielfach erwartet, als Tiefe. Gesteine mit niedriger Geschwindigkeit erscheinen in der Zeitsektion relativ mächtig, während Schichten mit hoher Geschwindigkeit ein relativ kleines Zeitintervall umfassen.

Aus rein mathematischer Sicht ist die Zeit-Tiefen-Konvertierung eine sehr einfache Aufgabe. Einzig notwendige Information ist die Ausbreitungsgeschwindigkeit akustischer Wellen in den einzelnen Gesteinspaketen, also die Intervallgeschwindigkeiten. Die Genauigkeit der Tiefenumrechnung ist folglich von der Genauigkeit der Intervallgeschwindigkeiten abhängig, weshalb diese Information bei Tiefensektionen möglichst genau ermittelt werden sollte.

Ist in der näheren Umgebung eines reflexionsseismischen Profils keine Bohrung vorhanden, gibt es nur relativ eingeschränkte Möglichkeiten, an Geschwindigkeitsdaten $\mathrm{zu}$ kommen. Das ist einerseits deren direkte Messung an Gesteinshandstücken und andererseits deren Berechnung aus den in der seismischen Datenauswertung gewonnenen Stapelgeschwindigkeiten. Dabei können abhängig von der Datenqualität Stapelgeschwindigkeiten mit einer Genauigkeit zwischen 5\% und $15 \%$ bestimmt werden. Zur Lithologieerkennung bilden Intervallgeschwindigkeiten ein ideales Hilfsmittel, da sie neben der Kompaktion direkte Funktionen der durchschallten Gesteine sind. 
Im Fall von zweidimensionalen Daten kann das wahre Schichteinfallen nur auf Linien genau in Einfallsrichtung oder aus mehreren sich kreuzenden Linien erkannt werden. Für die strukturelle Interpretation ist es weiters von Bedeutung, anstatt eines Zeitprofils ein adäquates unverzerrtes Tiefenprofil zur Verfügung zu haben. Von größerer Bedeutung ist daher die strukturelle Interpretation auf migrierten Sektionen, da nur in solchen alle nicht horizontalen Reflexionselemente in ihrer wahren Lage abgebildet sind. Einschränkend für die Migration ist allerdings, dass Reflexionen, die von seitlich außerhalb eines Profils stammen, nur in dreidimensionalen Datensätzen wieder ihrer tatsächlichen Raumlage zugeordnet werden können. Zudem benötigt man für die Migration genaue Geschwindigkeitsdaten. Im Inntal stehen solche Daten nicht im ausreichenden Maße zur Verfügung, weshalb auf die Migration verzichtet wurde.

Die seismofazielle Interpretation (Seismostratigraphie) ist ein Konzept, bei dem aus seismischen Fazieseinheiten (Sequenzen) Aussagen über die Entwicklung eines Sedimentbeckens, sedimentäre Strukturen und die lithologische Zusammensetzung gemacht werden. Voraussetzung für eine erfolgversprechende Anwendung ist ein Datenmaterial guter Reflexionsmächtigkeit mit möglichst enger Profildichte. Da diese Voraussetzungen beim derzeitigen Stand im Inntal noch nicht gegeben sind, können nur Ansätze zu einer solchen Analyse gemacht werden. Bei der Untersuchung diluvialer Ablagerungen sind nicht alle jene Parameter gleich bedeutend wie im klassischen Falle mariner Sedimentbecken. Folgende Faziesparameter spielen eine Rolle: Reflexionsform, Reflexionskontinuität, Reflexionsamplitude, Frequenz, Reflexionshäufigkeit, Reflexionsgeometrie, Reflexionskonfiguration, Intervallgeschwindigkeit.

Für den Erfolg der Reflexionsseismik als Untersuchungsmethode ist das Vorhandensein von Leithorizonten guter Reflexionsqualität und weiter Verbreitung eine wesentliche Voraussetzung. Ein solcher Leithorizont sollte erwartungsgemäß die Quartärbasis sein, da für den präquartären Untergrund Gesteine hoher Geschwindigkeit (Karbonate, kristalline Schiefer) anzunehmen waren.

Ein Leithorizont an der Quartärbasis (Q) scheint jedoch im Oberen Inntal nur lokal zu existieren. Dafür könnten zwei Ursachen verantwortlich sein: Zum einen könnte die Energieanregung $(0,21 \mathrm{~kg} /$ Schuss $)$ für die beträchtlichen Tiefen der Quartärbasis zu gering gewesen sein. Eine andere Möglichkeit könnte sein, dass an der Quartärbasis ein geringer Reflexionskoeffizient existiert. Dafür könnten die Ergebnisse des Sonic-log in der Tiefbohrung Wattens 1 sprechen, die Geschwindigkeiten von $4000 \mathrm{~m} / \mathrm{s}$ im tieferen Teil des 
Quartärs ergeben haben. Alleine zur Klärung dieser Frage ist eine weitere Messkampagne erforderlich, bei der die Grundlagenforschung im Vordergrund steht.

Aus den vorliegenden Profilen alleine ist es kaum möglich, eine regional gültige seismofazielle Analyse zu machen. Für eine solche sind auch die Ergebnisse der Reflexionsseismik aus dem Unterinntal, Zillertal und die Daten der Bohrungen Flughafen Innsbruck und Wattens $1 \mathrm{zu}$ berücksichtigen. Ein langgestreckter, flach liegender, erster Leithorizont (mit „O“ bezeichnet) dürfte eine Verlandungsserie sein. Die darunter liegenden Schichtglieder sind demnach als lakustrin aufzufassen. Eine reflexionsleere bis reflexionsarme Zone im Bereich bis zum Horizont A deutet auf feinkörnige Sedimente (Tone, Schluffe, Sande) hin. Im Horizont A ist die jüngste Übertiefung ausgebildet, wie das Profil Oberhofen 9001 zeigt. In der darunter liegenden Sequenz wird vorwiegend lakustrine Sedimentation angenommen, wobei einzelne Hinweise auf Deltabildungen gegeben sind. Geneigte Reflexionsgeometrie könnte für glaziofluviatile Sedimente sprechen, wobei die Reflektoren kurz, uneben und von geringer bis mäßiger Reflexionsstärke sind. Das Reflexionsbild im vermuteten basalen Bereich des Quartärs lässt auch an das Vorkommen von älterer Moräne bzw. Grundmoräne oder Blocklehme denken, was auch aufgrund der Intervallgeschwindigkeiten möglich wäre.

\subsection{Diskussion der Ergebnisse der einzelnen Messgebiete}

Völs

Der Beginn der Analyse der seismischen Daten des Oberen Inntals erscheint insofern mit Völs sinnvoll, da dieser Raum dem durch Tiefbohrungen besser bekannten Gebiet Flughafen Innsbruck am nächsten liegt; allerdings ist die Entfernung von ca. $3 \mathrm{~km}$ für eine Korrelation unter Umständen noch immer zu groß. Das $1,75 \mathrm{~km}$ lange Profil streicht $\mathrm{W} 10^{\circ} \mathrm{N}$ und erstreckt sich $0,6 \mathrm{~km}$ bis $0,8 \mathrm{~km}$ südlich des Inns. Es liegt weiters ca. $600 \mathrm{~m}$ vom südlichen Talrand entfernt, was für die Berücksichtigung von Seiteneinsätzen wesentlich ist.

Bei der Beurteilung der Reflexionsqualität ist deutlich ein Zusammenhang mit der Abnahme des Überdeckungsgrades gegen das westliche und östliche Profilende zu erkennen (Abb. 6). Es lassen sich aufgrund der erwähnten Kriterien beim ersten Processing zunächst zwei seismofazielle Einheiten abgrenzen. Die obere Einheit 


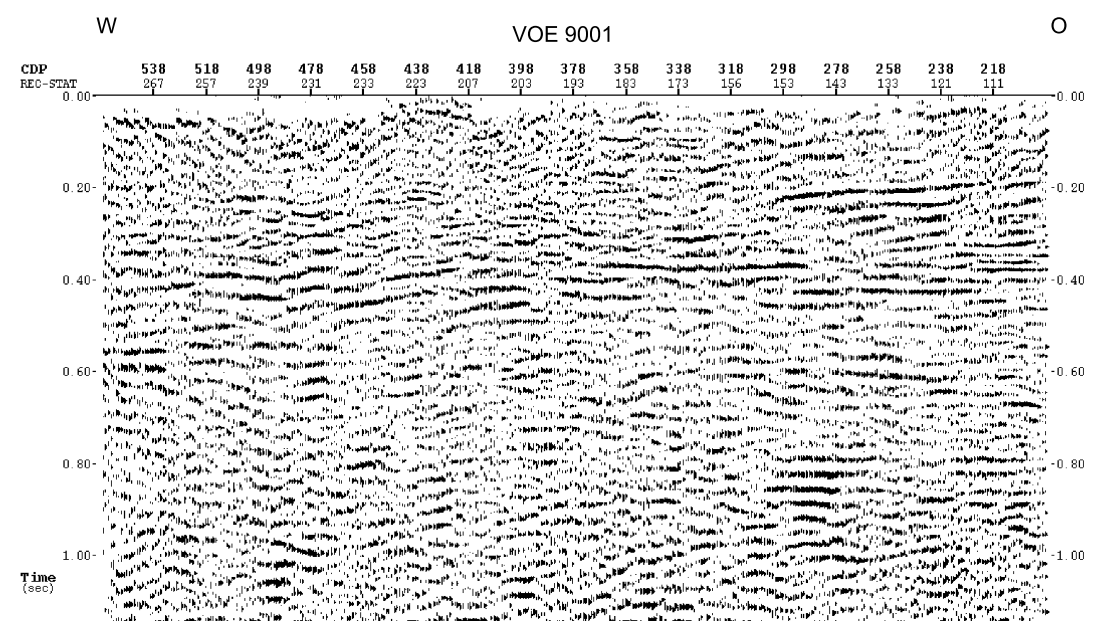

Abb. 6. Zeitprofil VOE9001 (Stand 1990)

erstreckt sich im Zeitbereich von ca. 0,2-0,4 s und hat vor allem gegen das Liegende eine klare seismische Abgrenzung, im Ostteil in Form eines langgestreckteren oberen Reflektors. Die Oberkante wird bis zur Station 154 durch einen Leithorizont gebildet, der jedoch gegen W abrupt endet. Die ganze Sequenz fällt bis dorthin ganz gegen $\mathrm{W}$ ein. Weiter nach $\mathrm{W}$ zu findet sich bis ca. $0,3 \mathrm{~s}$ herab eine nahezu chaotische Zone mit einer kleinen Muldenstruktur bei Station 232, die gegen das Liegende zu bis $0,3 \mathrm{~s}$ hinabreicht. Die Basisreflexion der oberen Sequenz zeigt im W bei teilweise guter Reflexionsamplitude Anklänge an eine dachziegelförmige Konfiguration. Eine eigenartige Reflexionskonfiguration tritt im Bereich von Station 142 bis 152 auf: Hier entsteht unter dem erwähnten Reflektor ein aus vier Phasen bestehendes Reflexionsband. Auch sonst treten unterhalb der Sequenz von $0,8 \mathrm{~s}$ vereinzelte, meist kurze Reflexionselemente auf, so im Abschnitt 122-158 bei ca. 1,0 s, ebenso lokal im Westteil. Es ist aber derzeit noch fraglich, ob es sich hierbei nicht um Reflexionselemente anderer Entstehung, etwa Einsätze von der Talflanke oder Processingrelikte, handelt.

Im Ostteil des Profils bildet ein tieffrequenter Reflektor (ca. $40 \mathrm{~Hz}$ ) bei etwa 0,4 s die Basis der Sequenz, wobei die Lagerung teils söhlig, teils schwach westfallend ist. In der tieferen Sequenz treten nur wenige Reflektoren längerer Erstreckung auf, so eine im Osten mit ca. $600 \mathrm{~m}$ Länge bei $0,6 \mathrm{~s}$, im W ein bis Station 235 reichender Horizont von $400 \mathrm{~m}$ Länge. Gemeinsam ist diesen Reflektoren, dass 


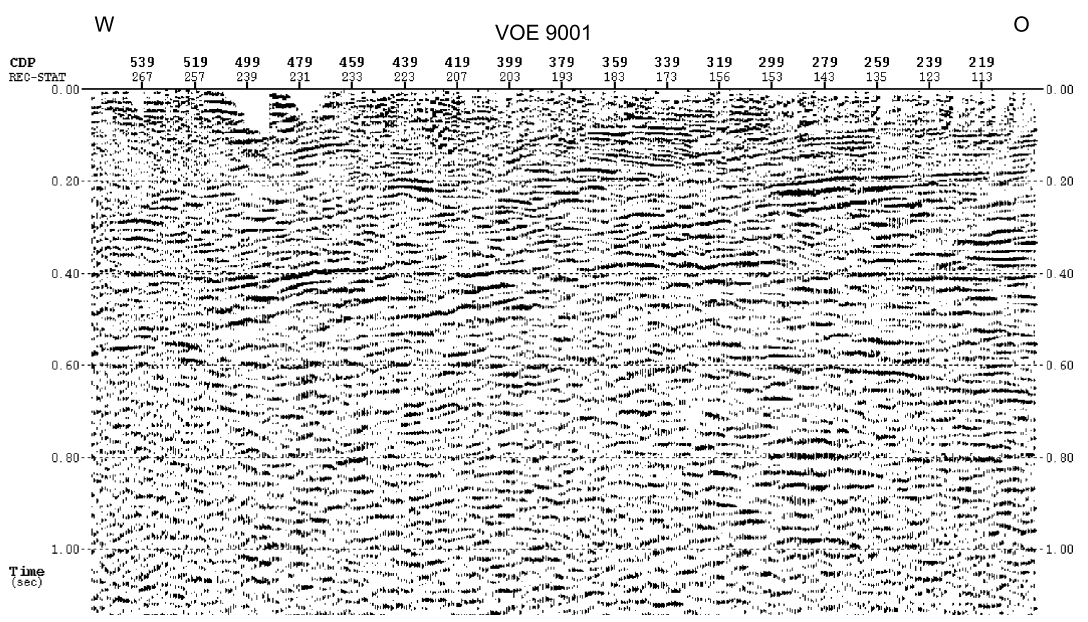

Abb. 7. Zeitprofil VOE9001, Reprocessing 2003

sie eine unebene Grenzfläche aufweisen. Auch einige kurze, gekrümmte Reflektoren zeichnen sich ab.

Das reprocesste Profil ist auf ein um $20 \mathrm{~m}$ höheres Bezugsniveau ( $+570 \mathrm{~m}$ ü.A.) bezogen, wodurch noch ganz seichte Reflexionen aus dem Talalluvium von ca. 0,03 s interpretiert werden konnten (Abb. 7). Im Zeitbereich bis 0,2 s wurden zahlreiche hochfrequente Reflektoren, die im alten Profil nur angedeutet waren, mit guter Amplitude und Reflexionscharakteristik herausgearbeitet. Das erleichtert auch die seismofaziellen Aussagen; das abrupte Ende des Reflektors bei Station 185, der sich im Zeitbereich von 0,1 s im ganzen Ostteil des Profils mit söhliger Lagerung erstreckt, hat sicher sedimentäre Ursachen. Andererseits weisen die Reflektoren bei ca. 0,4s eine andere Reflexionscharakteristik mit streckenweiser Kontinuität auf. Das westliche Einfallen im Abschnitt Station 215 bis 255 kommt deutlicher heraus. Der am alten Profil vermutete steilere Anstieg der Quartärbasis von Station 240 bis zum westlichen Profilende ist im reprocessten Profil nicht vorhanden. Als Quartärbasis könnten einige lokal stärker reflektierende Elemente bei $0,6 \mathrm{~s}$ angesehen werden. Unabhängig von der Datengewinnung dürfte der tiefere Teil des Quartärs reflexionsarm bis reflexionsleer sein.

Es wird folgende geologische Interpretation für zutreffend gehalten (Abb. 8): Im Westteil wird ein ca. $200 \mathrm{~m}$ tiefes, spät-postglaziales Seetonbecken mit feinklastischen Sedimenten vermutet, in das Lagen gröberklastischer Gesteine eingeschaltet sind. Die östliche Flanke wird teils durch nach $\mathrm{O}$ ansteigende Reflexionen, teils durch das 


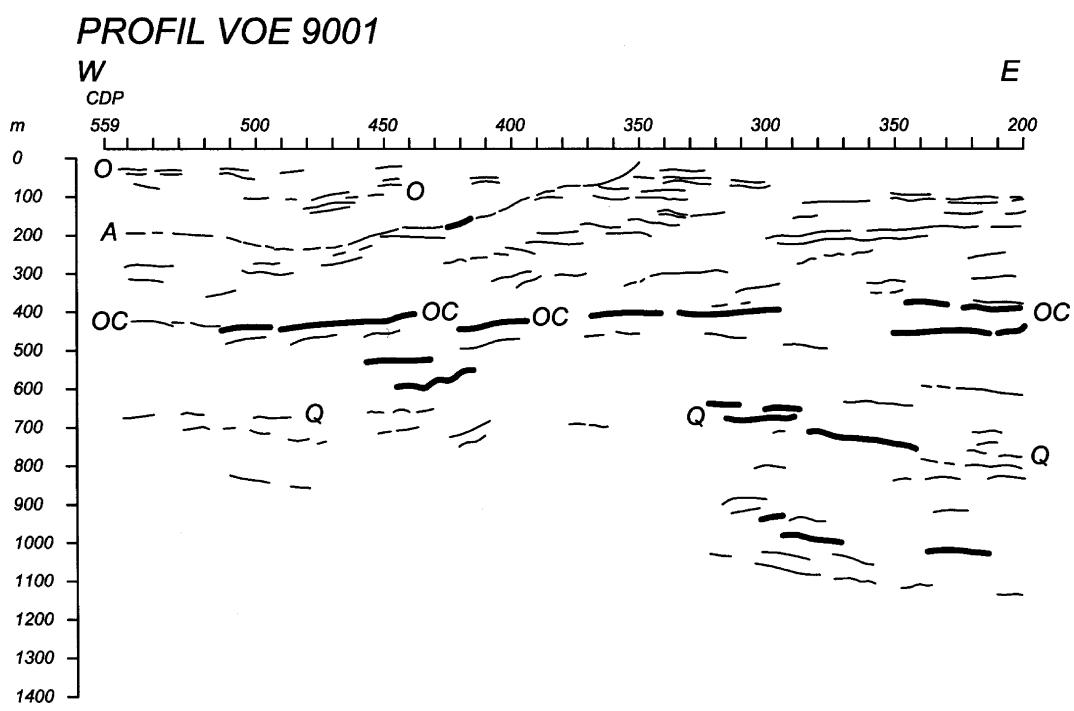

Abb. 8. Tiefenprofil VOE9001 und Interpretation

abrupte Enden von Reflexionen festgelegt. Damit ist die Basis (Horizont A) näherungsweise angegeben. Die Oberkante der überkompaktierten Schichten (Horizont OC) liegt im Tiefenbereich von ca. $400 \mathrm{~m}$ unter dem Datumniveau. Die Quartärbasis, falls deren Lage in ca. $700 \mathrm{~m}$ unter dem Bezugsniveau richtig ist, würde im östlichen Profilteil sehr flach nach $\mathrm{O}$ einfallen. Die Identität der zwischen Station 105-150 sich im Tiefenbereich von 950-1000 m unter BN erstreckenden Elemente ist derzeit noch ungeklärt.

\section{Oberhofen}

Dieses Messgebiet ist deshalb besonders wertvoll, da es als einziges zwei sich kreuzende Profile umfasst, wodurch die grundsätzlich verschiedenen strukturellen Verhältnisse klar zum Ausdruck kommen. Es belegt auch die Erkenntnis, dass der Bauplan übertiefter Täler ohne Querprofile nicht verstanden werden kann.

Das $1,53 \mathrm{~km}$ lange, nahezu N-S verlaufende Profil OH9001 ist durch eine markante, praktisch das ganze Profil ausfüllende Muldenstruktur im oberen Teil der Schichtfolge gekennzeichnet (Abb. 9). Die nördliche Flanke ist durch eine nahezu kontinuierliche Reflexion großer Amplitude und ebener Ausbildung hervorragend 


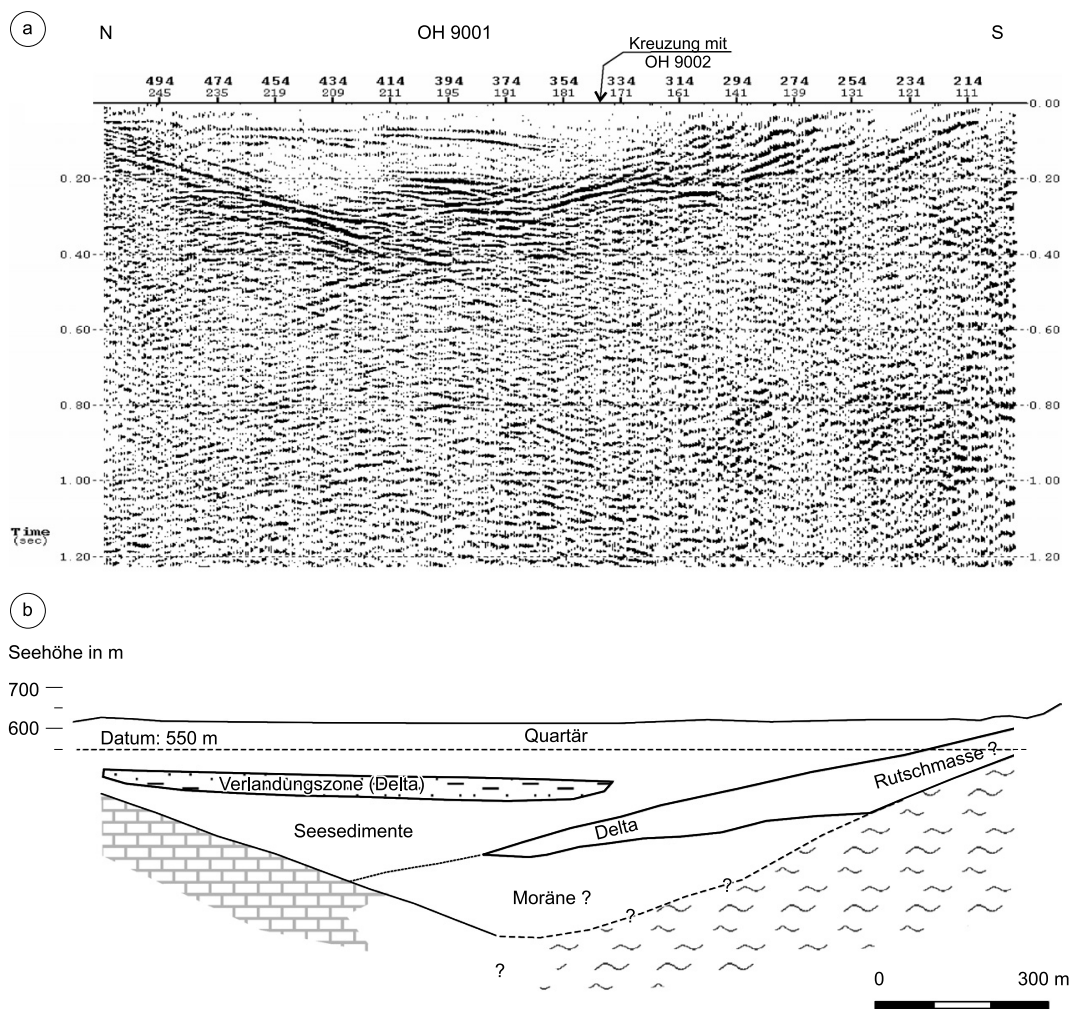

Abb. 9. Zeitprofil OH9001 (a) und geologische Interpretation (schematisches Tiefenprofil, b)

belegt. Das Zentrum der Mulde liegt bei Station 180 bei ca. $0,52 \mathrm{~s}$, knapp nördlich des Schnittes mit der Linie OH9002. Weniger gesichert ist der Verlauf des südlichen Schenkels der Mulde, weil sich dort kein durchgehender ebener Reflektor als Muldenbasis abzeichnet. Der Grund könnte im verminderten Impedanzkontrast als Folge in der unterschiedlichen Lithologie liegen, wobei im Norden Kalkalpin und im Süden Quarzphyllit anstehen.

Aufschlussreich ist auch die Reflexionskonfiguration innerhalb der Talfüllung. Im tiefsten Teil der Mulde und der Flanken treten einige kürzere, flach liegende Reflexionselemente auf. Diese könnten als ein Hinweis auf eine Grundmoräne gelten. Ein Paket von kräftigen Reflexionen ist zwischen St 185-205 zu erkennen, deren Lagerung man als Kreuzschichtung erkennen könnte. Daran schließt sich nach 
Süden ein mittelsteil ansteigender kräftiger Reflektor an, in dessen Hangende parallele, allerdings schwächere Reflexionen ausgebildet sind. Aufgrund des Kriteriums der Schichtneigung wird dies als Deltasediment klassifiziert. Die weitere südliche Fortsetzung ist durch eine andere Charakteristik, nämlich durch unebene, teils nach oben konvexe Reflektoren gekennzeichnet. Die Konfiguration in diesem Bereich kann als schräg bis dachziegelartig, vielleicht auch sigmoidal bezeichnet werden. Wenn letzteres zutrifft, dann würde dieses Paket den proximalen Anteil der Deltasedimente darstellen. Andererseits wäre wegen der Nähe zum südlichen Beckenrand auch an Rutschmassen zu denken. Am südlichsten Profilende tritt im Zeitbereich um $0,1 \mathrm{~s}$ ein kräftiger Reflektor auf, der mit der Oberkante des Quarzphyllits korreliert werden könnte.

Die Talfüllung ist im nördlichen Abschnitt von St 210 bis St 240 durch Reflexionsarmut bzw. -leere gekennzeichnet, was als Hinweis auf eine homogene feinklastische Sedimentation (,Seetonbecken“) gelten kann. Schwächere nach Norden ansteigende Reflexionen könnten von gröberklastischen Einschaltungen verursacht sein. Gegen das Hangende zu wird die Schichtfolge von einem kräftigen Reflektor abgeschlossen, der im Norden bei ca. 0,07 s söhlig liegt und sodann allmählich flach nach Süden einfällt und vor dem Schnitt mit Profil $\mathrm{OH} 9002$ endet. Es handelt sich hierbei um den Topbereich der Verlandungsserie. Es sind eindeutig toplaps knapp unterhalb dieses Horizontes ausgebildet, woraus eine Schüttung von Norden her abzuleiten ist.

Ein etwas anderes Bild zeigt das Profil OH9002 (Abb. 10). Es lassen sich auf diesem zwei Sequenzen unterscheiden, wobei sich im Querprofil OH9001 die innerquartären Reflexionen sicher korrelieren lassen. Die oberste Sequenz zeigt bei $0,07 \mathrm{~s}$ den Top, fällt also mit dem Abschluss der Mulde von OH9001 zusammen, wird also auch auf diesem Profil mit dem Top der Verlandungsserie korreliert. Die Basis der 1. Sequenz bildet eine kräftige Reflexion, die bei St 150 bei 0,17 s liegt und nach Osten bis ca. St 114 gleichförmig einfällt und sodann in eine flache Lagerung übergeht. Dieser Reflektor wird mit den als Deltasedimente im Profil OH9001 angesprochenen Reflexionen korreliert. Bei der Interpretation ist der räumliche Verlauf der Profile zu beachten. Im Hangenden sind mounded und downlapförmige Reflexionen zu erkennen. Das spricht für eine Einschüttung aus südlicher Richtung. Auch rezent mündet der aus Süden kommende Kanzingbach noch an dieser Stelle ins Inntal.

Die Unterkante der 2. Sequenz liegt bei St 150 in $0,51 \mathrm{~s}$ und fällt nach $\mathrm{O}$ bis auf $0,61 \mathrm{~s}$ bei St $114 \mathrm{ab}$. In geologischer Hinsicht dürfte 


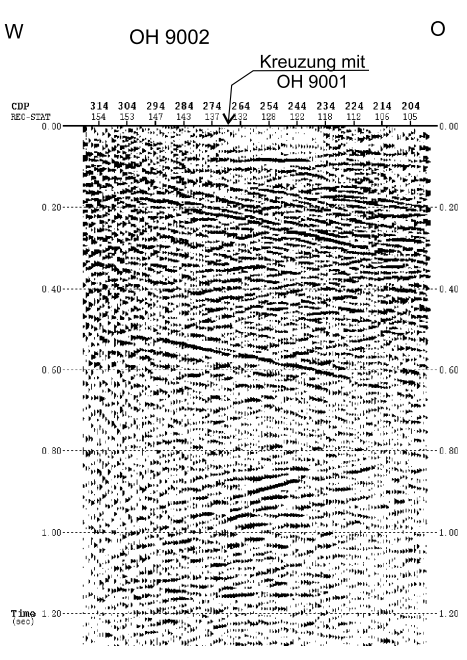

(a)

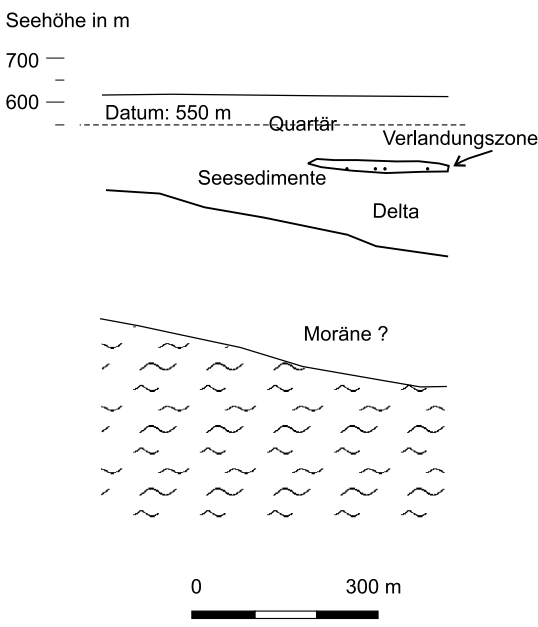

(b)

Abb. 10. Zeitprofil OH9002 (a) und geologische Interpretation (schematisches Tiefenprofil, b)

diese Reflexion der Quartärbasis entsprechen. Die Internkonfiguration der 2. Sequenz ist durch zahlreiche gegen W ansteigende, seltener flachliegende, kurze Reflektoren gekennzeichnet; dabei ist in einigen Fällen ersichtlich, dass diese von Sequenz 1 abgeschnitten werden. Andererseits zeigen Reflektoren ein downlap gegen die Quartärbasis.

Der tieferliegende Bereich ist durch weitgehende Reflexionsarmut charakterisiert, was für die Interpretation als präquartärer Untergrund spricht.

\section{Silz}

Das Profil Silz streicht WSW-ONO, ist also ein Längsprofil (Abb. 11). Auffallendstes Merkmal ist ein aus zwei Reflektoren bestehendes Reflexionsband im Zeitbereich von $0,18-0,25 \mathrm{~s}$. Dieses verläuft absätzig, hat jedoch zwischen Stationen 165-135 einen längeren ebenen Reflektor. Zwischen den Stationen 190-225 deutet sich eine flach-kuppelförmige Lagerung an, ebenso bei St 180. Im östlichen Teil herrscht deutlich ein Einfallen gegen Osten. Im oberflächennahen Bereich lassen sich vereinzelt söhlige, schwache Reflexionen bei 0,09 s erkennen. Im Abschnitt St 210-195 steigt ein Reflektor mit 


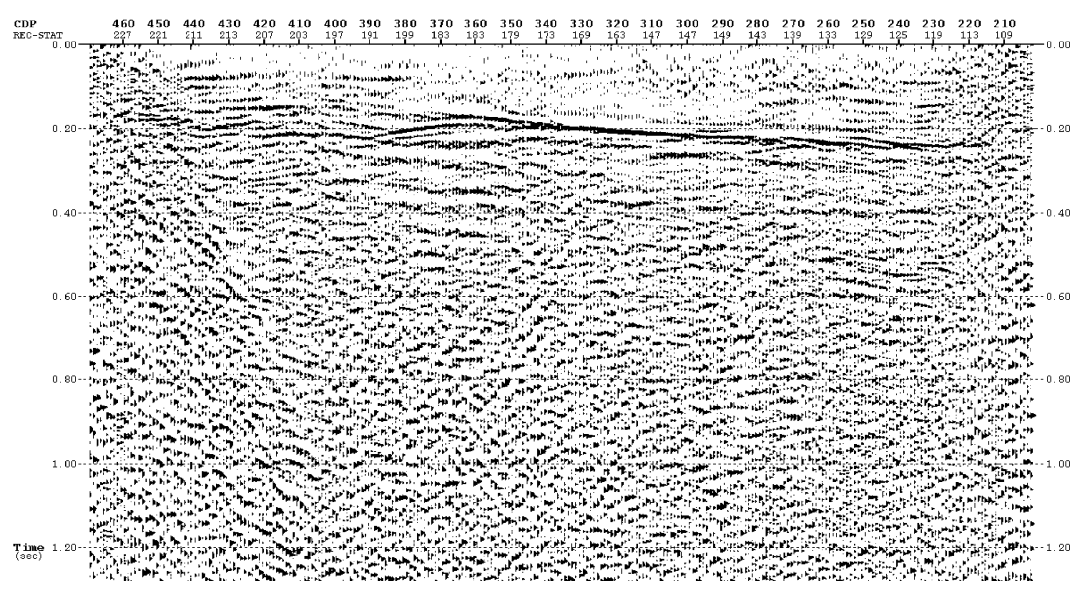

Abb. 11. Zeitprofil SI9001

Laufzeit von $0,31 \mathrm{~s}$ gegen Osten bis an den im Hangenden sich erstreckenden Hauptreflektor heran. Ansonsten sind in dieser 2. Sequenz im Laufzeitbereich bis ca. $0,65 \mathrm{~s}$ vereinzelte schwache Reflektoren mit unebenem Verlauf ausgebildet. Neben wenigen söhlig liegenden Elementen fallen diese generell gegen ONO ein. Ein ca. $150 \mathrm{~m}$ langes Reflexionsband erstreckt sich im Abschnitt St 130-115 im Zeitbereich von 0,50-0,64 s. Darunter treten zwar auf kurze Distanz einzelne, schwächere Elemente auf, einwandfreie Reflexionshorizonte, vor allem solche, die man als tiefliegende Quartärbasis ansprechen könnte, fehlen jedoch.

\section{Refraktionsseismik Mils}

Das refraktionsseismische Profil Mils (Abb. 12) erstreckt sich im Inntal westlich der Pitzmündung knapp nördlich des Inns. Das $1200 \mathrm{~m}$ lange Profil streicht WSW-ONO und verläuft somit spitzwinkelig zum heutigen Verlauf des Flusses. In seismischer Hinsicht handelt es sich um einen 3-Schichtfall. Unter einer geringmächtigen Oberflächenschicht trockener Lockersedimente mit variabler Geschwindigkeit von $540-750 \mathrm{~m} / \mathrm{s}$ liegt die Hauptmasse des grundwasserführenden Quartärs, dessen Geschwindigkeit mit $2220 \mathrm{~m} / \mathrm{s}$ praktisch konstant ist. Der $\mathrm{V}_{3}$-Refraktor ist durch hohe Geschwindigkeiten von $5740 \mathrm{~m} / \mathrm{s}$ gekennzeichnet, die den dichten 


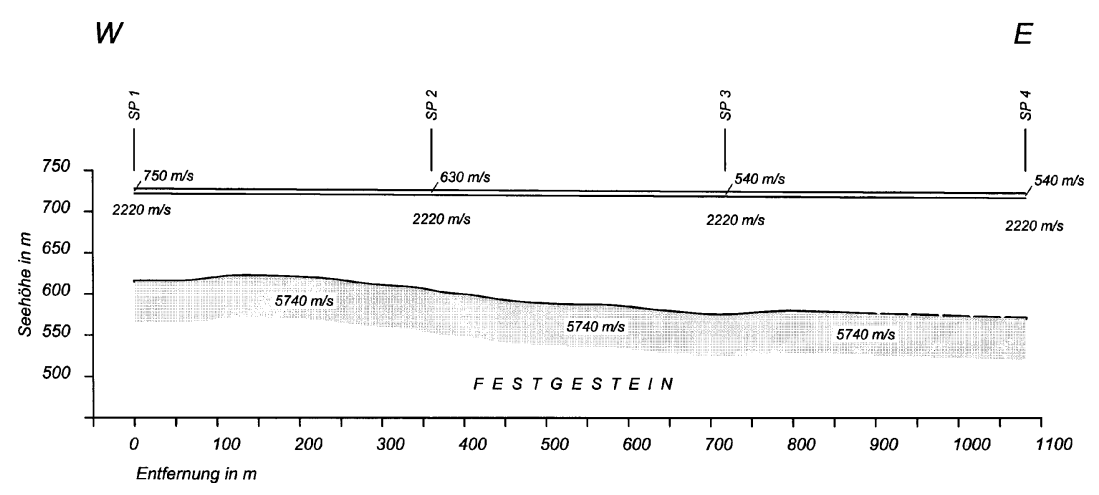

Abb. 12. Refraktionsseismik Mils 9001

Karbonaten des präquartären Untergrundes entsprechen, von dem aufgrund der Geschwindigkeiten angenommen werden kann, dass er unverkarstet sein muss. Das Profil erstreckt sich ca. 200 (im E)-300 m (im W) nördlich der anstehenden Triaskarbonate. Das Profil zeigt ein deutliches Relief des Untergrundes, dessen Tiefe nach ONO auf ca. $150 \mathrm{~m}$ zunimmt. Die einheitliche Geschwindigkeit der Beckenfüllung lässt den Schluss zu, dass keine Leithorizonte größerer Geschwindigkeit ausgebildet sind.

\subsection{Seismofazielle Analyse}

Eine seismofazielle Analyse aus den Daten des Messgebietes Oberes Inntal alleine ist nur mit großen Einschränkungen möglich, was auf das weitgehende Fehlen von Talquerprofilen und die kurze Profillänge zurückzuführen ist. Der Versuch einer Erkennung bestimmter seismofazieller Muster kann jedoch bei voller Anerkennung seines hypothetischen Charakters unternommen werden, wenn ergänzende Daten einbezogen werden durch:

- Reflexionsseismische Ergebnisse aus dem Unteren Inntal

- Geologisches Profil der Bohrung Wattens 1 einschließlich der Bohrlochgeophysik

- Geologische Profile der Bohrungen vom Flughafen Innsbruck

- Geschwindigkeitsmodelle

Die reflexionsseismischen Daten in 5 Messgebieten des Unteren Inntals zeigen durchwegs gute Qualität, wobei einzelne Reflexions- 
bänder durchaus den Charakter von Leithorizonten erreichen (WEBER und SCHMID, 1991). Schwierigkeiten bereitete die eindeutige reflexionsseismische Festlegung der Quartärbasis. Als Geometrie des übertieften Tals konnte im Raum Wattens eine asymmetrische Trogform nachgewiesen werden.

Das geologische Profil der Tiefbohrung Wattens 1 weist nach VINZENZ (1990) eine Grobgliederung in einen oberen Bereich $(0-350 \mathrm{~m})$ und einen unteren Bereich (350-900 m) auf, wobei letzterer überkonsolidierte Sedimente umfasst. Die Bohrung verblieb bis zur Endteufe im Quartär. Der obere Bereich umfasst unkonsolidierte Sande, Kiese und Schotter mit einer mächtigen Seetonlage. Der untere Bereich besteht aus lehmig-schluffig gebundenen Klastika mit vereinzelten reinen Schotterlagen. Die Grenze bei $350 \mathrm{~m}$ Teufe muss eine bedeutende Faziesgrenze darstellen, wofür die starke Zunahme des Tongehalts (Gammaray-log), ein Dichtesprung von 2,5 auf ca. $2,8 \mathrm{~g} / \mathrm{cm}^{3}$ und ein Geschwindigkeitssprung von $2400 \mathrm{~m} / \mathrm{s}$ auf $4000 \mathrm{~m} / \mathrm{s}$ sprechen. Nach MOSTLER (1982) wurden in der Tiefbohrung Flughafen Innsbruck in 140 bis $240 \mathrm{~m}$ Teufe eine Folge von Sand, Kies und Ton erbohrt, darunter bis zur Endteufe von $368,5 \mathrm{~m}$ fast ausschließlich Sand; bemerkenswert ist die Angabe im Bohrmeisterprofil von ,fester Lagerung bzw. Moräne“ ab 174 m Teufe (POSCHER, 1992).

Die in regelmäßigen Abständen auf den Profilen durchgeführte Geschwindigkeitsanalyse ist eine wichtige Voraussetzung für die lithologische Interpretation. Die Genauigkeit hängt allerdings von der Reflexionsqualität und der Länge der Reflektoren ab und nimmt generell mit zunehmender Laufzeit ab. Die Angabe der Durchschnitts- und Intervallgeschwindigkeiten auf Einerstellen genau darf nicht darüber hinwegtäuschen, dass dies ein reiner Rechenwert aufgrund des Processingalgorithmus ist. Die Geschwindigkeitswerte des Bereichs unter ca. $0,5 \mathrm{~s}$ und über etwa $3000 \mathrm{~m} / \mathrm{s}$ geben nur die Größenordnung an. Davon ist auch eine stellenweise auftretende Geschwindigkeitsumkehr betroffen, deren Realität in jedem Fall durch Vergleich mit der Qualität der Originaldaten hinterfragt werden muss.

Folgende seismofazielle Einheiten können beim derzeitigen Stand der Untersuchungen wahrscheinlich gemacht werden:

Moräne und Blocklehm: Kurze, meist tieffrequente Reflexionselemente, auch mit unebener Fläche; die Reflexionsmuster im Muldenbereich von Profil $\mathrm{OH} 9001$ könnten dafür sprechen, die Geschwindigkeiten dürften über $3000 \mathrm{~m} / \mathrm{s}$ betragen. 
Glaziofluviatile Sedimente: Mäßige Reflexionsqualität, variable Längserstreckung, lokal leicht schräge Reflexionskonfiguration, unebene Lineamente sprechen für grobklastische, wenig geschichtete Sedimente.

Turbidite: Oftmals gute Reflexionsqualität, Reflexionserstreckung variabel, Auftreten leicht konvexer Konfiguration, stellenweise größere Reflexionsdichte. Beispiel: Westteil von Profil SI 9001.

Deltabildungen: Hauptkriterium ist die Schichtneigung, befriedigende Reflexionsqualität, Erstreckung unterschiedlich lang, Geschwindigkeitsbereich unter $2500 \mathrm{~m} / \mathrm{s}$. Beispiel: Westteil von Profil OH 9002 zwischen 0,2 bis $0,5 \mathrm{~s}$.

Glaziolakustrine Ablagerungen: Gute Reflexionsqualität und Längserstreckung, oftmals hohe Reflexionsdichte. Geschwindigkeiten variabel je nach Tiefenlage. Auftreten auf allen Profilen.

Seetone: Auftreten im oberen Teil der Talfüllung; meist reflexionsleere Abfolge von feinklastischen Sedimenten (Ton, Schluff); einzelne schwache, flach liegende Reflektoren stammen von Kieslagen. Geometrie der Seetonbecken durch kräftige Basisreflexion, abruptes Aufhören von Reflexionen an den Rändern charakterisiert, lokal auch Verzahnung an den Rändern. Niedrige Geschwindigkeiten unter $1900 \mathrm{~m} / \mathrm{s}$. Gegen das Hangende Abschluss durch längeres Reflexionsband, flach liegend, gute Reflexionsqualität. Beispiel: Profil OH 9001, OH 9002, SI 9001.

Massenbewegungen, Rutschmassen: Kurze, auch kräftige geneigte Reflexionselemente, die im Flankenbereich auftreten; absätzige Reflexionskonfiguration, downlap-ähnliche Reflexionsmuster. Beispiel: Südrand von Profil OH 9001.

Talalluvium: Niederfrequente, flach liegende diskontinuierliche Reflexionselemente dürften den Flussschottern entsprechen.

\section{Zwischenbilanz über den Bauplan des Oberen Inntals}

Die wenigen, nicht zusammenhängenden Profile machen es schwierig, ein klares Bild vom Bauplan des Oberen Inntals zu entwerfen. Wenn das einzige Querprofil bei Oberhofen repräsentativ ist, dann scheint jedenfalls kein U-förmiges Trogtal bei der Übertiefung vorzuliegen. Das Längsprofil des Oberen Inntals lässt ein komplexes Relief des präquartären Untergrundes vermuten (Abb. 13). Sicher ist das 


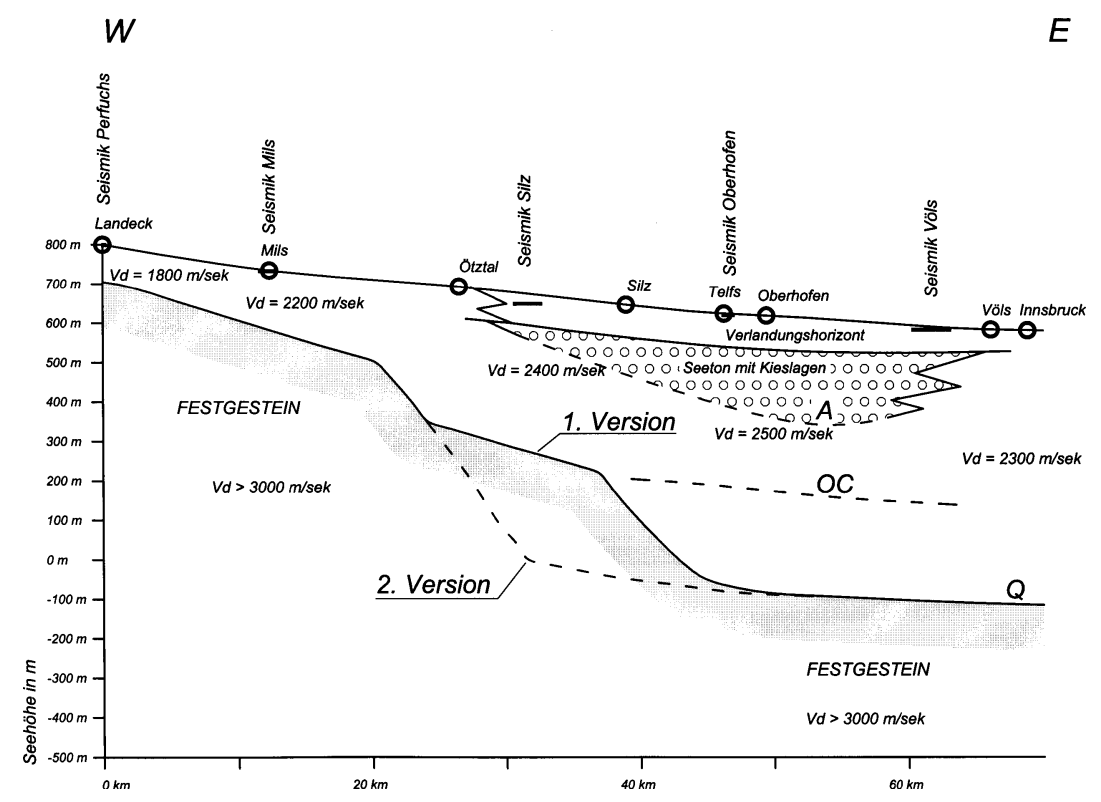

Abb. 13. Längsprofil des Oberen Inntales, Abschnitt Landeck-Innsbruck

mittelsteile östliche Einfallen des Untergrundes zwischen Landeck und Mils. Selbst wenn man im Raum Mils die erste Version bezüglich der Lage des Untergrundes annimmt und das Einfallen bei Mils weiter nach $\mathrm{O}$ extrapoliert, so ergibt sich westlich der Ötztalmündung eine Abtreppung um ca. $150 \mathrm{~m}$. Da im Abschnitt Oberhofen-Völs nur ein geringer Abfall in Tallängsrichtung aufscheint, ist eine weitere beträchtliche Stufe westlich Telfs notwendigerweise anzunehmen. Bei der zweiten Version ist ein weiterer schwacher Anstieg des Untergrundes bis in den Raum westlich Silz wahrscheinlich, worauf gegen W etwa bei der Ötztalmündung eine große Rampe von einigen $100 \mathrm{~m}$ folgen müsste. Überkompaktierte Schichten mit Geschwindigkeiten über $3000 \mathrm{~m} / \mathrm{s}$ konnten nur im Bereich großer Quartärmächtigkeiten (Abschnitt Völs-Oberhofen) nachgewiesen werden. Die Grenze der Überkompaktion dürfte nicht überall scharf sein, da die Intervallgeschwindigkeiten auch „Blindzonen“ mit einer Geschwindigkeitsabnahme anzeigen. Erhebliche Mühe wurde der Frage gewidmet, ob zwischen Silz und Völs ein zusammenhängendes Seetonbecken vorliegt, wie dies in Abb. 13 dargestellt ist. Ein lokales Auskeilen in Längsrichtung, wie es bei Völs und Oberhofen auf den Profilen möglich ist, könnte auch durch Einflüsse von der Talflanke her 
verursacht werden. Gegen das Hangende wird die als Seetonbecken postulierte Struktur durch einen in 50 bis $70 \mathrm{~m}$ Tiefe liegenden Verlandungshorizont reflexionsseismisch abgeschlossen. Völlig unsicher ist die Natur der im unteren Teil der quartären Schichtserie auftretenden, meist kurzen, auch unebenen Reflektoren. Es könnte sich um Reste älterer Moränen oder um Blocklehme handeln. Im Raum Oberhofen weisen die starken Schichtneigungen auf Deltasedimente hin, allerdings fehlen sigmoidale Reflexionsmuster, auch die genaue räumliche Lage ist noch ungeklärt.

\section{Ausblick}

Mit den geophysikalischen Messungen im Oberen Inntal wurde mehr noch als im Unteren Inntal Neuland betreten, da keine Tiefenaufschlüsse zur Eichung der reflexionsseismischen Daten zur Verfügung standen. Die vorliegenden Aussagen sind daher notwendigerweise nur ein Statusbericht, der mit Zunahme des Datenmaterials an Aussagekraft gewinnt. Das zeigt sich zwanglos bei einem Vergleich der Länge der Profile zum Talverlauf: Bei einer Länge des Inntals von Innsbruck bis zur Ötztalmündung ist nur eine Strecke von $3,65 \mathrm{~km}$, das sind ca. 9\%, von reflexionsseismischen Längsprofilen erfasst.

Eine wichtige Fragestellung ist die Datenqualität und deren mögliche Steigerung. Während im höheren Schichtstapel (in den Zeitprofilen bis etwa $500 \mathrm{~ms}$ ) die sedimentären Verhältnisse gut abgebildet werden, kann dies für zunehmende Laufzeit nicht vorbehaltlos angenommen werden, da die Primärenergie bei den vorliegenden Messungen relativ gering war. Der präneogene Untergrund ist vielfach von stark kompaktierten Sedimenten überdeckt, sodass der Impedanzkontrast an dieser Schichtgrenze niedrig ist. Nur schwache kurze Reflexionen werden erwartet, deren Terminationen die Präneogenoberkante nicht eindeutig definieren.

Bei einer Fortsetzung der Untersuchungen sollten daher die Messungen mit kräftigeren Energiequellen ausgeführt werden. Idealerweise sind dies Vibratoren oder zumindest an die Dämpfung in oberflächennahen Schottern angepasste gesteigerte Lademengen.

Für die Erkundung der Morphologie des Untergrunds und der Talfüllung sind Querprofile möglichst bis zum anstehenden Grundgebirge unerlässlich. Aus Sicht der Geophysik spricht alles für die Anlage eines systematischen Netzes von Längs- und Querprofilen. In Anbetracht der subtilen geologischen Situation wäre eine 3D-Seismik Stand der Technik, was jedoch wegen der hohen Kosten in nächster Zukunft nicht in Frage kommen dürfte. 
Generell muss auf die Fortschritte in der Aufnahmetechnologie hingewiesen werden, die sich, ähnlich wie im Processing, entwickelt haben. Neue Untersuchungen werden aber in jedem Fall von der vollen Zusammenführung vielfältiger Geodaten profitieren. Gravimetrie kann dabei als flächendeckende Begleituntersuchung und für Detailmessungen durchaus empfohlen werden, und zur Lithologieerkennung vor allem im oberen Stockwerk wären geoelektrische Untersuchungen sehr nützlich. Bohrlochgeophysik und digitale Geländemodelle sind weitere Informationsquellen. Daraus in einer integrierten Interpretationsmethodik abgeleitete geologische Modelle kommen schließlich den tatsächlichen Verhältnissen am nächsten.

\section{Danksagung}

Die vorliegende Arbeit beruht auf den Unterlagen des Projektes „Geophysik Oberes Inntal“, das aus Mitteln des Landes Tirol und des Bundesministeriums für Wissenschaft und Forschung durchgeführt wurde. Für die zur Verfügungstellung der Daten und die gute Zusammenarbeit danken die Autoren bestens. Das Reprocessing und die Manuskriptverarbeitung erfolgten mit Unterstützung der Forschungsgesellschaft Joanneum Research, wofür der Geschäftsleitung verbindlich gedankt wird. Weiters wird Frau M-Eng. C. WOHLFAHRT für die englische Übersetzung, Frau W. KoINIG für die Manuskripterstellung und Herrn G. HAUER für die Anfertigung von Abbildungen gedankt.

\section{Literatur}

[1] AMPFERER, O. (1921) Über die Bohrung von Rum bei Hall in Tirol und quartäre Verbiegungen der Alpentäler. Jb. Geol. B.-A. 71: 71-84

[2] ARIC, K., SteinhauSER, P. (1976) Geophysikalische Untersuchung des Inntaluntergrundes bei Thaur, östlich von Innsbruck. Zschr. Gletscherkunde u. Glaz. Geol. 12: 37-54

[3] BADER, K. (1979) Extarationstiefen würmeiszeitlicher und älterer Gletscher in Südbayern. Eiszeitalter und Gegenwart 29: 49-61

[4] EBERLE, M. (1987) Zur Lockergesteinsfüllung des St. Galler und Liechtensteiner Rheintales. Eclogae geol. Helv. 80/1: 193-206

[5] FincKH, P., KelTS, K., LAMBERT, A. (1984) Seismic stratigraphy and bedrock forms in perialpine lakes. Geol. Soc. of Amer. Bull. 95: 1118-1128

[6] FINCKH, P., FREI, W. (1991) Seismic reflection profiling in the Swiss Rhone valley. Eclogae geo. Helv. 84/2: 345-357

[7] FincKh, P., KLINGELE, E. (1991) Seismic reflection profiling in the Swiss Rhone valley, Part 2: Gravimetric and geological interpretation of the Roche-Vouvry line. Eclogae geol. Helv. 84: 359-368

[8] HeIssel, W. (1954) Beiträge zur Quartärgeologie des Inntales. Jb. geol. B.-A. 97: $251-322$

[9] VAN HusEN, D. (1979) Verbreitung, Ursachen und Füllung glazial übertiefter Talabschnitte an Beispielen in den Ostalpen. Eiszeitalter und Gegenwart 29: 9-22 
[10] Kissling, E., Schwendener, H. (1990) The Quarternary sedimentary fill of some Alpine valleys by gravity modelling. Eclogae geol. Helv. 83/2: 311-321

[11] MostleR, H. (1982) Kurze Stellungnahme zur Lockergesteinsbohrung Flughafen Innsbruck. In: INGERLE (Hrsg.) Erkundung der Grundwasserverhältnisse im Raum Innsbruck durch eine Tiefbohrung - Endbericht (unveröff. Gutachten). Tiroler Rohstoffforschungs- und Studiengesellschaft m.b.H.

[12] Patzelt, G. (1987) Untersuchungen zur nacheiszeitlichen Schwemmkegel- und Talentwicklung in Tirol. Veröffent. Mus. Ferd. 67: 93-123

[13] Patzer, U., PröHL, ST. (1988) Was ist seismische Stratigraphie? Teil 1: Methodische Grundlagen. Z. f. Angew. Geologie 34/11: 321-325; Teil 2: Anwendungsmöglichkeiten. Z. f. Angew. Geologie 34/12: 359-364

[14] Pfiffner, O. A., Heitzmann, P., Lehner, P., Frei, W., Pugin, A., Felber, M. (1997) Incission and backfilling of Alpine valleys: Pliocene, Pleistocene and Holocene processes. In: Pfiffner, O. A., Lehner, P., Heitzmann, P., Mueller, S., Steck, A. (eds.) Deep Structure of the Swiss Alps. Results of NRP 20: 265-288, Birkhäuser, Basel

[15] Posch, E., WalaCH, G. (1989) Das Bouguerschwerefeld in Vorarlberg und im Bereich der Übergangszone zwischen West- und Ostalpen. Österr. Beitr. z. Met. u. Geophysik 2: 147-152

[16] Posch, E., Walach, G. (1990) Das Bouguer-Schwerefeld in Vorarlberg. Mitt. Österr. Geol. Ges. 82: 105-118

[17] Poscher, G., SCHMID, C. (1992) Geologische Interpretation der seismischen und gravimetrischen Untersuchungen im Quartär des Oberen Inntales. Rohstoffforschungsprojekt TA/190 - Geophysik Oberes Inntal (unveröff. Forschungsbericht). Amt der Tiroler Landesreg., Kulturbauamt

[18] Pugin, A., RosetTI, S. (1992) Acquisition of land based high resolution seismic profiles in glacial basins, two case studies in the Alpine foreland of Switzerland. Eclogae geol. Helv. 85/2: 491-502

[19] RoKSANDIC, M. M. (1978) Seismic facies analysis concepts. Geophys. Prospect 26: $383-398$

[20] Schmid, C., Kogler, A., Stadlober, K., Hartmann, G. (1992) Ergebnisse der seismischen Messungen im Quartär des Oberen Inntales (unveröff. Bericht). Institut für Angew. Geoph., Joanneum Research, Leoben

[21] SAngree, J. B., Widmier, J. M. (1979) Interpretation of depositional facies from seismic data. Geophysics 44: 131-160

[22] Schoop, R. W., Wegener, H. (1984) Einige Ergebnisse der seismischen Untersuchungen aus dem Bodensee. Bull. Ver. Schweiz. Petrol.-Geol. u. Ing. 50/118: 55-61

[23] SHERIFF, R. E. (1980) Seismic Stratigraphy. Int. Human Resources Devel. Corp., Boston

[24] VAN HuSEN, D. (1979) Verbreitung, Ursachen und Füllung glazial übertiefter Talabschnitte an Beispielen in den Ostalpen. Eiszeitalter und Gegenwart 29: 9-22

[25] VinZENZ, M. (1990) Geologische und sedimentpetrographische Beschreibung der Bohrungen Wattens I, Wörgl I und Reutte I (unveröff. Projektbericht). Joanneum Research, Leoben

[26] WalaCh, G. (1990) Schwerkraftmessungen in Vorarlberg. Kulturinformation Vorarlberger Oberland 2: 77-82

[27] WeBER, F., SCHMID, CH. (1991) Reflexions- und refraktionsseismische Messungen im Zillertal und deren quartärgeologische Aussagen. Mitt. österr. Geol. Ges. 84: $205-221$ 
[28] Weber, F., Schmid, Ch., Figala, G. (1990) Vorläufige Ergebnisse reflexionsseismischer Messungen im Quartär des Inntals/Tirol. Ztschr. f. Gletscherk. u. Glazialgeol. 36/2: 121-144

[29] WILDI, W. (1984) Isohypsenkarte der quartären Felstäler in der Nord- und Ostschweiz, mit kurzen Erläuterungen. Eclogae geol. Helv. 77/3: 541-551

[30] Wright, V. P., MARRIOTT, S. B. (1993) The sequence stratigraphy of fluvial depositional systems: The role of floodplain sediment storage. Sed. Geol. 86: 203-320

[31] YILMAZ, Ö. (1987) Seismic data processing. Soc. Expl. Geophys. Tulsa, Oklahoma

Anschrift der Verfasser: Dipl.-Ing. Dr. Wilfried Gruber, Institut für Wasser RessourcenManagement - Hydrogeologie und Geophysik, Joanneum Research, Roseggerstraße 17, 8700 Leoben, Österreich; Prof. Dr. Franz Weber, Institut für Geophysik, Montanuniversität, Peter-Tunner-Straße 25, 8700 Leoben, Österreich. 